\title{
MINI-REVIEW
}

\section{Endothelial Cell Calcium Signaling during Barrier Function and Inflammation}

\author{
Prarthana J. Dalal, William A. Muller, and David P. Sullivan
}

From the Department of Pathology, Northwestern University Feinberg School of Medicine, Chicago, Illinois

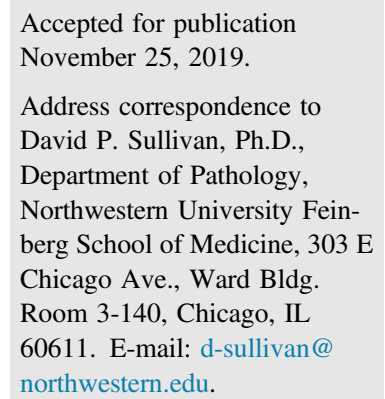

\begin{abstract}
Calcium is an essential second messenger in endothelial cells and plays a pivotal role in regulating a number of physiologic processes, including cell migration, angiogenesis, barrier function, and inflammation. An increase in intracellular $\mathrm{Ca}^{2+}$ concentration can trigger a number of diverse signaling pathways under both physiologic and pathologic conditions. In this review, we discuss how calcium signaling pathways in endothelial cells play an essential role in affecting barrier function and facilitating inflammation. Inflammatory mediators, such as thrombin and histamine, increase intracellular calcium levels. This calcium influx causes adherens junction disassembly and cytoskeletal rearrangements to facilitate endothelial cell retraction and increased permeability. During inflammation endothelial cell calcium entry and the calcium-related signaling events also help facilitate several leukocyte-endothelial cell interactions, such as leukocyte rolling, adhesion, and ultimately transendothelial migration. (Am J Pathol 2020, 190: 535-542; https://doi.org/10.1016/j.ajpath.2019.11.004)
\end{abstract}

\section{Common Molecules and Mechanisms in Calcium Signaling}

Endothelial cells serve diverse functions in health and disease. They form a crucial interface between blood and tissue and therefore are responsible for controlling permeability, regulating inflammation, balancing coagulation, and leading angiogenesis among many other roles. Calcium is a crucial second messenger, and many endothelial cell functions rely heavily on changes in intracellular calcium ions and the resultant signaling cascades. Quiescent endothelial cells at rest maintain a very low intracellular cytosolic free calcium concentration, ranging from 30 to $100 \mathrm{nmol} / \mathrm{L}$, and there is an approximately 20,000-fold concentration gradient across the plasma membrane. ${ }^{1,2}$ Low intracellular calcium concentrations are actively maintained by transmembrane channels expressed in the plasma membrane, such as the plasma membrane calcium ATPase channels, and by transmembrane channels in the endoplasmic reticulum (ER) membrane, such as the sarco/ER Ca ${ }^{2+}$-ATPase channels ${ }^{3}$ (Figure 1).

Calcium signaling can be stimulated in endothelial cells by inflammatory mediators, such as thrombin, histamine, and bradykinin. The intracellular calcium level increases 5 to 10 times over baseline when these mediators bind their receptors, which include G-protein-coupled receptors or receptor tyrosine kinases. Agonist-occupied G-protein-coupled receptors or activated receptor tyrosine kinases subsequently activate phospholipase $\mathrm{C}$ which then facilitates the hydrolysis of the phospholipid phosphatidylinositol 4,5-bisphosphate to produce diacylglycerol and inositol-1,4,5-trisphosphate. Inositol-1,4,5-trisphosphate in turn induces a calcium influx from intracellular calcium stores within the ER (Figure 1). Inositol-1,4,5trisphosphate-mediated ER $\mathrm{Ca}^{2+}$ release results in intracellular store depletion, which subsequently initiates $\mathrm{Ca}^{2+}$ release-activated $\mathrm{Ca}^{2+}$ entry from the extracellular space through store-operated cation channels. ${ }^{4}$ Transient receptor potential canonical channel 1 , the predominant transient receptor potential channel expressed in human endothelial cells, is an example of a store-operated cation channel. ${ }^{5}$ Store-operated calcium entry can also be mediated by Stim1 and Orai1. Stim1 senses low calcium concentrations

Supported by NIH grants R01 HL046849 (W.A.M.), R01 HL064774 (W.A.M.), T32GM8152 (P.J.D.), and F30HL134202 (P.J.D.).

Disclosures: None declared. 


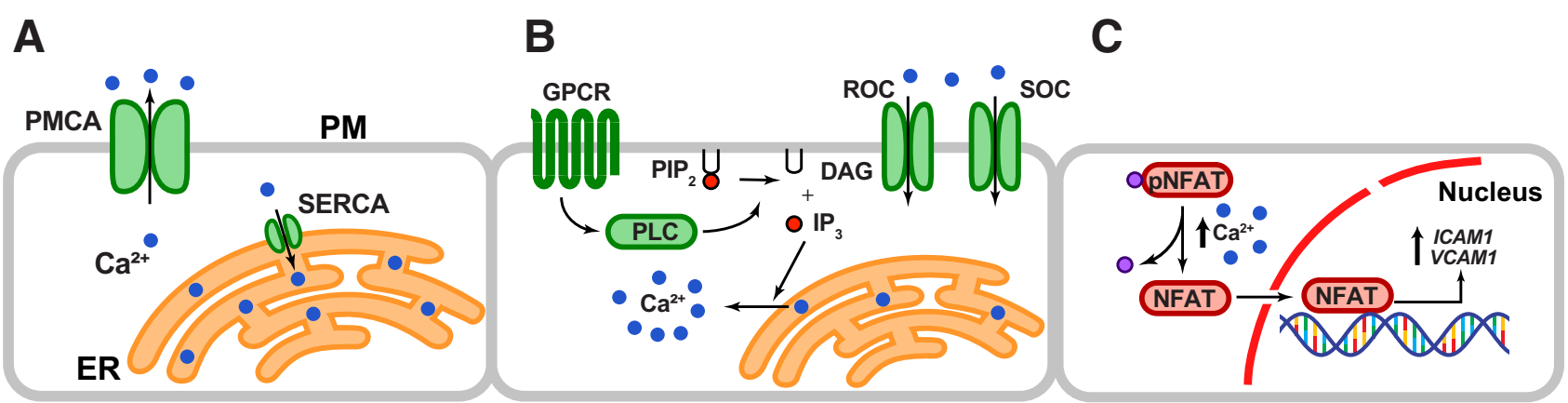

Figure 1 A: Low intracellular calcium concentrations at rest are maintained by plasma membrane calcium ATPase (PMCA) and sarco/endoplasmic reticulum (ER) $\mathrm{Ca}^{2+}$-ATPase (SERCA) channels. PMCA channels are present on the plasma membrane (PM), and SERCA channels are present on the ER membrane. Both actively transport calcium ions out of the cytoplasm and into the extracellular space and ER, respectively, to maintain low intracellular calcium concentrations. B: On stimulation of G-protein-coupled receptors (GPCRs), phospholipase C (PLC) is activated, resulting in the hydrolysis of phosphatidylinositol 4,5bisphosphate $\left(\mathrm{PIP}_{2}\right)$ to diacylglycerol (DAG) and inositol triphosphate $\left(\mathrm{IP}_{3}\right) . \mathrm{IP}_{3}$ in turn induces calcium influx from the ER and extracellular space. Calcium entry from the extracellular space can occur from store-operated cation channels (SOCs) or receptor-operated cation channels (ROCS). C: During inflammation, the calcium influx into endothelial cells can facilitate dephosphorylation of nuclear factor of activated T-cells (NFAT) from phospho-NFAT (pNFAT) to NFAT. NFAT then travels to the nucleus, where it can increase transcription of ICAM1 and VCAM1.

in the ER and activates Orai1 to increase intracellular $\mathrm{Ca}^{2+}$ and replenish ER calcium stores. Stolwijk et $\mathrm{al}^{6}$ reported that the effects of thrombin and histamine on endothelial permeability can be mediated by STIM1.

Calcium entry from the extracellular space can also occur through receptor-operated cation channels, which can be directly activated without prior depletion of ER calcium stores. For example, transient receptor potential channel 6 (TRPC6), which is responsible for endothelial calcium influx as it relates to leukocyte transendothelial migration (TEM), is activated by diacylglycerol and is an example of a receptor-operated cation channel. ${ }^{7,8}$ Typically, intracellular $\mathrm{Ca}^{2+}$ store release is associated with a transient increase in calcium, whereas $\mathrm{Ca}^{2+}$ entering from the extracellular space classically produces sustained calcium entry. ${ }^{5}$ Ultimately, the increase in endothelial intracellular calcium then triggers a number of diverse signaling pathways.

Calcium influx is detected by proteins that contain specialized $\mathrm{Ca}^{2+}$-binding motifs. These effector proteins help couple the fluctuations in intracellular calcium to affect a variety of cellular functions, including $\mathrm{Ca}^{2+}$ buffering in the cytoplasm, signal transduction, and gene expression. Generally, $\mathrm{Ca}^{2+}$-binding proteins can be characterized into 3 categories: EF-hand proteins, $\mathrm{C} 2$ domain proteins, and annexins.

EF-hand domains are the most common $\mathrm{Ca}^{2+}$-binding motif among proteins. This motif is named after the $\mathrm{E}$ and $\mathrm{F}$ helices in the protein parvalbumin, where this domain was first described. ${ }^{9}$ EF hands contain a helix-loop-helix structure that cradles the calcium ion within an amino acid loop. The affinity of EF hand domains for calcium ions can vary substantially based on the identities of the amino acids present within the calcium-binding loop. ${ }^{1}$ Although the amino acid sequence within the loop may vary, the EF hands are structurally conserved with seven negatively charged, typically carboxylate functional groups that facilitate the $\mathrm{Ca}^{2+}$ binding. Examples of EF-hand $\mathrm{Ca}^{2+}$-binding proteins include troponin $\mathrm{C}$, parvalbumin, S100 proteins such as S100A7 and S100A8, calpain, and calmodulin $(\mathrm{CaM}) .{ }^{10}$ Proteins with a high affinity for calcium, such as parvalbumin $\left(\mathrm{K}_{\mathrm{d}}\right.$ of approximately $\left.10 \mathrm{nmol} / \mathrm{L}\right)$, serve as buffering proteins and work to maintain calcium homeostasis by adjusting the duration and shape of the $\mathrm{Ca}^{2+}$ signal. The structures of these buffering proteins remains similar regardless of whether they are in their $\mathrm{Ca}^{2+}$-free or $\mathrm{Ca}^{2+}$-bound forms. Other $\mathrm{Ca}^{2+}$-binding proteins with lower affinity, such as $\mathrm{CaM},\left(\mathrm{K}_{\mathrm{d}}\right.$ of approximately $\left.10 \mu \mathrm{mol} / \mathrm{L}\right)$, serve to sense physiologically relevant changes in intracellular calcium and translate this to cellular function. This process is accomplished through conformational changes in structure induced by $\mathrm{Ca}^{2+}$ binding. When $\mathrm{Ca}^{2+}$ is bound to these sensor proteins, their structure opens substantially to allow interaction with downstream targets. ${ }^{9}$

$\mathrm{CaM}$ is a $17-\mathrm{kDa}$ protein composed of four EF-hand motifs that are connected with a flexible helical linker. ${ }^{11}$ $\mathrm{CaM}$ is shaped like a dumbbell, and in humans it is transcribed from three different and independent loci. Despite differences in the genomic sequences of these three loci, they all produce transcripts that are translated into the identical protein. A single CaM polypeptide can bind up to four $\mathrm{Ca}^{2+}$ ions. $\mathrm{CaM}$ exists as both apoCaM (without $\mathrm{Ca}^{2+}$ bound) and $\mathrm{Ca}^{2+}$-bound CaM. ${ }^{12}$ Although $\mathrm{Ca}^{2+}$-bound $\mathrm{CaM}$ can exist with one to four calcium ions bound at varying combinations of the four sites, typically three or four $\mathrm{Ca}^{2+}$ ions are required to be bound before $\mathrm{Ca}^{2+}-\mathrm{CaM}$ can activate downstream targets. ${ }^{13}$ Although a substantial portion of total $\mathrm{CaM}$ engages with downstream targets in the $\mathrm{Ca}^{2+}$-bound form, apoCaM also plays a crucial regulatory role within cells and can bind proteins reversibly or irreversibly. For example, apoCaM can bind voltage-gated $\mathrm{Ca}^{2+}$ channels to up-regulate opening and, after binding calcium, provides feedback inhibition to reduce channel opening. ${ }^{14}$ This type of strategic pre-association of apoCaM with proteins can have significant implications for their 
spatiotemporal regulation by $\mathrm{Ca}^{2+} \cdot{ }^{15}$ In several cases, apoCaM helps create a feedback loop that allows protein complexes to be primed so that the cellular responsiveness to a calcium influx can be accelerated.

$\mathrm{CaM}$ has no intrinsic enzymatic activity, and its primary function is to amplify the calcium signal. As a result of $\mathrm{Ca}^{2+}$ binding to $\mathrm{CaM}$, a large conformational change occurs such that the hydrophobic surfaces of $\mathrm{CaM}$ are exposed. These hydrophobic surfaces allow $\mathrm{CaM}$ to interact with downstream proteins, such as substratespecific CaM kinases [CaMKIII, phosphorylase kinase, and myosin light chain kinases (MLCKs)] or multifunctional CaM kinases (CaMKI, CaMKII, and CaMKIV). ${ }^{11}$ Substrate-specific CaM kinases have a dedicated function within the cells in which they are expressed because their activity is typically restricted to one downstream target protein. On the other hand, multifunctional $\mathrm{CaM}$ kinases have several downstream targets, allowing them to affect many diverse cellular pathways. The presence of IQ motifs on target proteins can also help enhance the ability of $\mathrm{CaM}$ and other members of the EF-hand family to bind. IQ motifs are composed of the consensus sequence IQXXXRGXXXR and are present in a variety of proteins, including IQ motif-containing GTPase-activating protein 1 , neuromodulin, and myosin IIa.

CaMKII in particular has a role in affecting endothelial barrier function. For example, thrombin increases CaMKII activation, and inhibiting CaMKII activation with KN-93 attenuates thrombin-mediated increases in endothelial permeability. ${ }^{16}$ Furthermore, targeted knockdown of CaMKII $\delta$ also significantly attenuates increases in endothelial permeability in response to thrombin. ${ }^{17}$

S100 family proteins are another example of EF-hand proteins. Similar to $\mathrm{CaM}, \mathrm{Ca}^{2+}$ binding to $\mathrm{S} 100$ proteins allows hydrophobic surfaces to be exposed, thus facilitating interactions with target proteins. S100 proteins often act as intracellular regulators and extracellular signaling proteins. They assist in coordinating a wide range of cellular processes, such as angiogenesis, inflammation, cellular proliferation, and migration. In microvascular endothelial cells specifically, S100A8 is induced by proinflammatory stimuli. ${ }^{18}$ S100A8 and S100A9 induce F-actin disorganization in human umbilical vein endothelial cells and induce monolayer permeability through the p38 and ERK1/2 signaling pathways. ${ }^{19}$

Another family of proteins that can bind $\mathrm{Ca}^{2+}$ include $\mathrm{C} 2$ domain proteins. These proteins contain $\mathrm{C} 2$ domains that are composed of a $\beta$-sandwich structure and can typically bind two or three $\mathrm{Ca}^{2+}$ ions in the variable loops that connect the two $\beta$-sheets. When calcium is bound within this loop, the protein's affinity for lipids increases, facilitating the translocation of these proteins to specific areas of the membrane. C2 domain proteins are often key signal transduction regulators and include proteins such as phospholipases, protein kinase $\mathrm{C}$ (PKC), and phosphoinositide 3-kinase. ${ }^{1}$ Consequentially, $\mathrm{C} 2$ domain proteins help link
$\mathrm{Ca}^{2+}$ signaling with membrane functions, such as exocytosis, endocytosis, and ion flux regulation across membranes.

Similar to $\mathrm{C} 2$ domain proteins, annexins also use $\mathrm{Ca}^{2+}$ binding to facilitate their association with negatively charged phospholipid membrane surfaces. When bound to calcium, annexins also dock onto negatively charged membrane surfaces to facilitate membrane-related events. ${ }^{20}$ Within endothelial cells, annexin A2 is the most wellstudied member of the annexin family. Annexin A2 has been reported to support vascular integrity within the pulmonary microvasculature. Microvascular endothelial cells deficient in annexin A2 exhibit reduced barrier function. ${ }^{21}$ Annexin A2-deficient mice develop more substantial vascular leak, pulmonary edema, and neutrophil infiltration in response to alveolar injury compared with control mice. ${ }^{21}$ Annexin A2 can bind to actin and bundle actin filaments through residues on its C-terminus, and it also interacts directly with VE-cadherin in endothelial cells. Its ability to connect the VE-cadherin complex to cholesterol rafts and actin filaments helps strengthen adherens junctions as endothelial cell monolayers mature. ${ }^{22}$ This mechanism further supports a critical role for annexin A2 in maintaining endothelial barrier function.

\section{Localized Calcium Signals and Barrier Function}

Impaired endothelial barrier function is implicated in the morbidity and mortality associated with a number of pathologic conditions, including sepsis, pulmonary edema, and reperfusion injury. Without an adequate vascular endothelial (VE) barrier, the balance of water and protein between intravascular and extravascular compartments is disrupted. Endothelial cells can be connected by three types of junctions: adherens junctions, tight junctions, and gap junctions. ${ }^{23}$ Adherens junctions mediate cell-cell contact and are primarily composed of homophilic VE-cadherin interactions between adjacent endothelial cells. Tight junctions, composed of claudins and occludins, function to restrict the paracellular flux of fluid and are especially prominent in the cerebral vasculature of the blood brain barrier. Although gap junctions do not directly contribute to the endothelial barrier, they are important for mediating cell-cell communications and are composed of connexin family proteins that form pores between adjacent cells.

Calcium is a crucial second messenger involved in the signaling pathways that affect endothelial permeability. During an acute injury, altered barrier function occurs from the release of inflammatory mediators by the injured tissue and recruited leukocytes. These inflammatory mediators result in an intracellular calcium influx. Examples of inflammatory mediators include vasoactive amines, such as histamine and serotonin; peptides, such as bradykinin; the protease thrombin; and eicosanoids, such as thromboxanes, leukotrienes, and prostaglandins. During states of 
inflammation, these mediators bind receptors on endothelial cells that trigger channel opening, resulting in increased intracellular calcium concentration and activation of calcium-dependent proteins. ${ }^{5}$

Endothelial cell TRPC6 regulates vascular permeability as induced by inflammatory mediators. ${ }^{24,25}$ TRPC6 is a nonselective cation channel that is five times more permeable to $\mathrm{Ca}^{2+}$ than $\mathrm{Na}^{+}$at voltages near reverse potential. In endothelial cells, TRPC6 forms homotetramers. Each of the subunits have six membrane-spanning domains and a pore domain that lies between the fifth and sixth transmembrane domains. TRPC6 can be activated directly by diacylglycerol, mechanical stretch, and exposure to reactive oxygen species. ${ }^{26}$ TRPC6 also plays a role in mediating bradykinin and thrombin-induced permeability, further suggesting that endothelial cell calcium influx through TRPC6 is crucial during inflammation for increasing permeability. ${ }^{25,27}$ Numerous studies have examined how specifically this endothelial cell calcium influx facilitates increases in permeability. This increase in permeability occurs through disassembly of adherens junctions and rearrangement of cytoskeletal elements facilitating endothelial cell retraction. ${ }^{28}$

In particular, endothelial permeability changes induced by thrombin have been well studied. Although thrombin is a serine protease perhaps best known for its central role in the coagulation cascade, it plays a critical function in inflammation, particularly in situations in which the endothelium is disrupted and/or the coagulation cascade is activated. Thrombin acts through proteinase-activated receptor 1 (PAR-1), a G-protein-coupled receptor. Thrombin binds to PAR-1 and cleaves its N-terminus to generate a new amino terminus that affects signaling (Figure $2 \mathrm{~A}$ ). ${ }^{29}$ In terms of barrier function, thrombin binding PAR-1 mediates

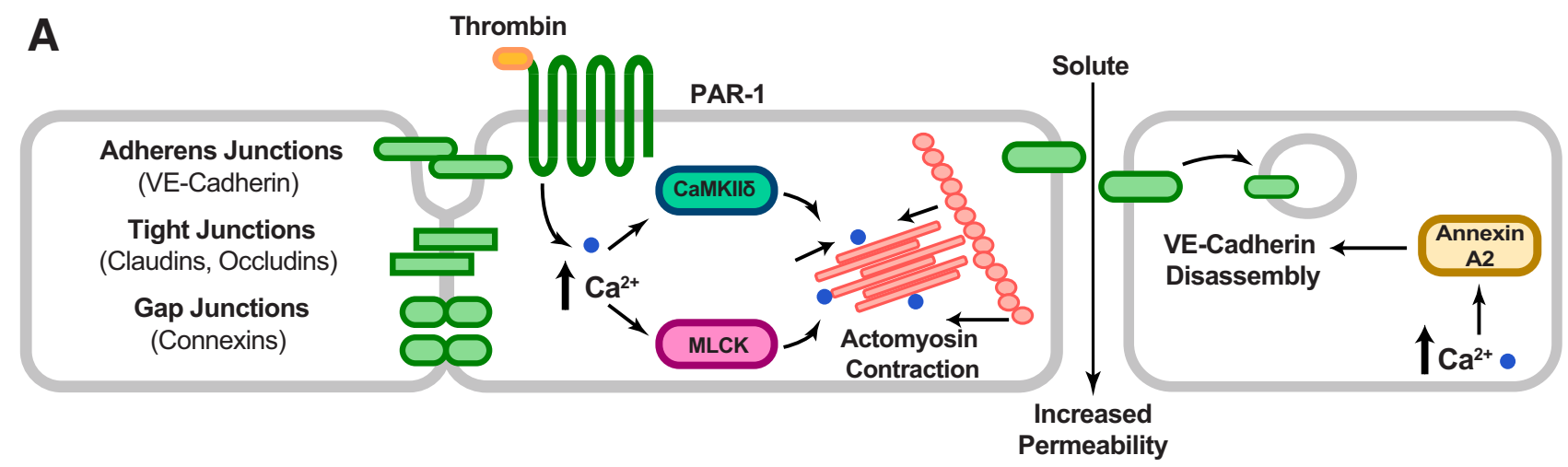

B

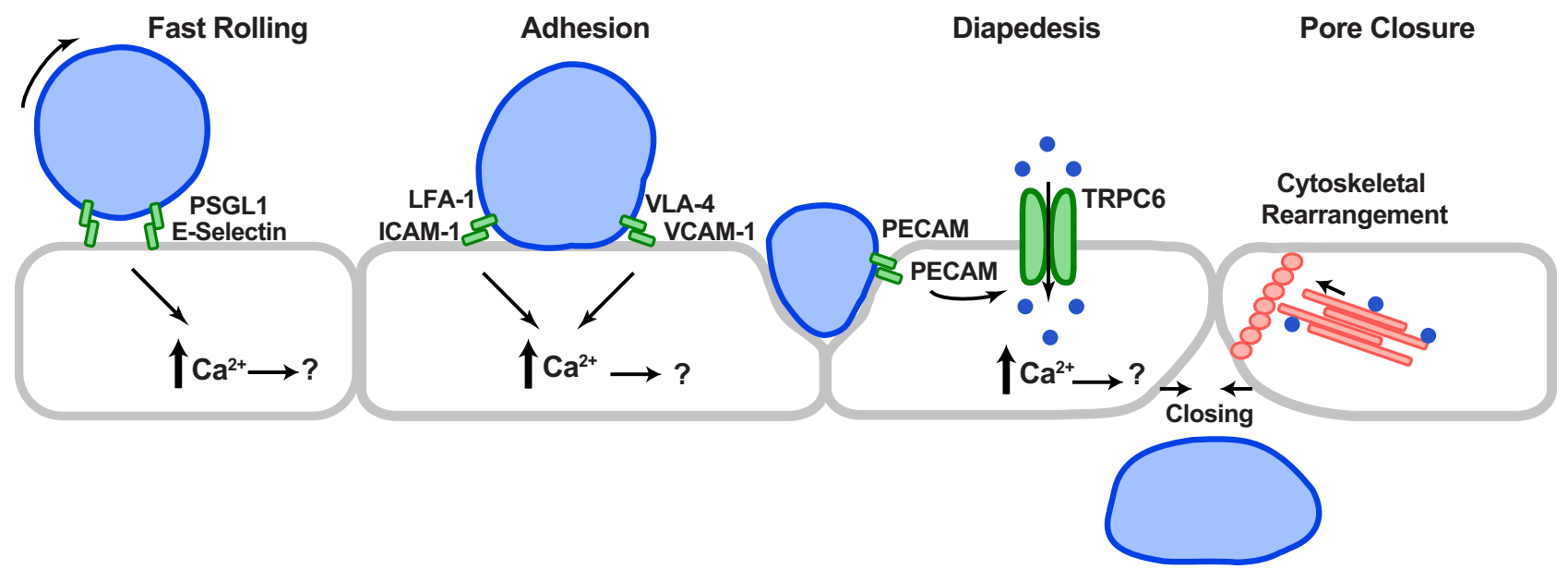

Figure 2 A: Endothelial calcium and barrier function. Thrombin binds a G-protein-coupled receptor (GPCR), PAR-1, resulting in an intracellular increase in $\mathrm{Ca}^{2+}$. This activates CaMKII $\delta$, myosin light chain kinase (MLCK), and annexin A2. Together these molecules facilitate vascular endothelia (VE)-cadherin disassembly and actomyosin contraction, leading to increased permeability. B: Endothelial calcium and inflammation. Endothelial calcium is a key regulator for many steps of the extravasation cascade. During fast rolling, endothelial E-selectin engagement mediates an increase in intracellular calcium concentration. As leukocytes adhere, they use lymphocyte function-associated antigen 1 (LFA-1) and very late antigen 4 (VLA-4) to interact with endothelial surface intercellular adhesion molecule 1 (ICAM-1) and vascular cell adhesion molecule 1 (VCAM-1). Both of these interactions promote increased intracellular calcium. Then during transendothelial migration, homophilic platelet endothelial cell adhesion molecule (PECAM)-PECAM interactions stimulate transient receptor potential channel 6 (TRPC6), resulting in a calcium influx necessary to support diapedesis. Finally, calcium ions are required to activate myosin light chain, which facilitates cytoskeletal rearrangements during the pore closure step. PSGL1, P-selectin glycoprotein ligand 1. 
increases in permeability. Mice deficient in the PARl gene had significantly attenuated increases in permeability in response to thrombin as measured by capillary permeability coefficient and pulmonary artery pressure. ${ }^{30}$ Furthermore, transient receptor potential channel 1-deficient mice also have significantly reduced responses to thrombin, further suggesting that thrombin signaling in the context of endothelial barrier function is calcium dependent. ${ }^{31}$ One way thrombin affects barrier function is by inducing VEcadherin disassembly. VE-cadherin disorganization leads to the loss of functional adherens junctions, thereby weakening the endothelial cell barrier. Thrombin-induced VE-cadherin disassembly is thought to occur through PKC activation. Inhibiting PKC using inhibitors such as calphostin $\mathrm{C}$ prevented VE-cadherin disassembly induced by thrombin. $^{32}$

Treating human umbilical vein endothelial cells with thrombin or thapsigargin (which induces release of ER-store $\mathrm{Ca}^{2+}$ ) results in increased intracellular calcium, activation of PKC, and decreased transendothelial monolayer electrical resistance, a measure of permeability. ${ }^{33}$ Treating endothelial cells with ionomycin, a calcium ionophore, also increases permeability. Specifically, treatment with thapsigargin and thrombin cause the translocation and activation of PKC $\alpha$ and disassembly of the VE-cadherin adherens junctional complex, suggesting that in addition to thrombin, the mechanism for thapsigargin-induced permeability also relies on activation of PKC and the resultant VE-cadherin junction disassembly. ${ }^{33}$

Actin-myosin engagement and cytoskeletal contraction also increase endothelial permeability. Under baseline conditions, the endothelial barrier is maintained with a balance between contractile and adhesive forces. However, as intracellular calcium increases in response to an inflammatory stimulus, MLCK is activated. MLCK phosphorylates myosin light chains (MLCs), such as $\mathrm{MLC}_{20}$, to promote their interactions with actin. This process leads to cytoskeletal contractile forces predominating over the adhesive forces.

Histamine, another proinflammatory mediator, also induces a $\mathrm{Ca}^{2+}$-dependent increase in endothelial permeability. Histamine-induced increases in permeability can be inhibited by chelating intracellular calcium with BAPTA-AM. Inhibiting CaM activity with trifluoperazine or inhibiting MLC phosphorylation with ML-7 also prevents histamine-induced increases in permeability. ${ }^{34}$

Loss of barrier function due to histamine was also associated with VE-cadherin disassembly. ${ }^{35}$ VE-cadherin phosphorylation and disassembly weakens adherens junctions so that endothelial cells can pull apart, ultimately forming paracellular gaps that increase permeability. ${ }^{36}$ Taken together these findings suggest that histamine relies on calcium signaling through $\mathrm{CaM}$ and MLC to mediate VE-cadherin junction disassembly and cytoskeletal rearrangement, ultimately resulting in decreased barrier function and increased endothelial permeability.
Ultimately, additional in vivo validation of calcium signaling pathways is needed to complement many of these in vitro studies. Future research should explore how calcium signals can affect endothelial barrier function in a contextdependent manner and how the pathways can vary based on a stimulus, vascular bed, or disease state.

\section{Differences in Endothelial Barrier Function in Various Vascular Beds}

Endothelial barrier function also varies by blood vessel type and tissue, raising the question of how endothelial calcium signaling and barrier function are locally coordinated in various vascular beds. For example, arteries and the blood brain barrier have well-developed tight junctions and therefore low permeability, whereas postcapillary venules, capillaries, and the endothelium of the liver have higher baseline permeability due to weaker intercellular contacts and the presence of fenestrations, respectively. This contrast is further evidenced by a study that found that tumor necrosis factor $\alpha$ treatment can increase permeability in the murine inferior vena cava but not in the murine aorta. ${ }^{37}$ Even within an individual organ there may be significant variation among subsets of endothelial cells. For example, pulmonary microvascular endothelial cells express calcium channels, such as the vanilloid family transient receptor potential 4 channel and the $\alpha 1 \mathrm{G}$ T-type calcium channel, which are not found in extraalveolar endothelial cells. ${ }^{38}$ This finding suggests that rather than taking a panendothelial approach to studying calcium signaling, it may be important to study endothelial calcium signaling in a more anatomically restricted and context-dependent fashion.

Furthermore, it is critically important to understand how calcium signaling is spatially controlled within endothelial cells. Recent evidence suggests that physiologic endothelial $\mathrm{Ca}^{2+}$ signaling may be dynamic, and localized signals as well as precise targeting of effector proteins may be key for $\mathrm{Ca}^{2+}$-dependent regulation of endothelial functions. For example, endothelial transient receptor potential 4 channel can produce $\mathrm{Ca}^{2+}$ transients localized along plasma membranes within mouse mesenteric artery endothelium. These signals can couple to nearby $\mathrm{K}_{\mathrm{Ca}}$ channels, causing hyperpolarization and generating positive feedback to augment the original $\mathrm{Ca}^{2+}$ signal. ${ }^{39}$ Although the advances in highspeed confocal imaging have markedly improved the ability to record spatiotemporally diverse $\mathrm{Ca}^{2+}$ signals, much complexity still remains. Developing quantitative approaches to comprehensively characterize $\mathrm{Ca}^{2+}$ activity in endothelial cells, particularly in the context of barrier function and inflammation, still remains a challenge. Although the field has made several advancements in using global $\mathrm{Ca}^{2+}$ measurements and treatments, future studies will likely need to shift focus to analyzing more local profiles of $\mathrm{Ca}^{2+}$ dynamics in response to distinct stimuli. Furthermore, the study of live calcium dynamics in vivo has 
also been relatively limited. Although many strategies have focused on evaluating isolated arterial segments and using cell-permeant fluorescent dyes, such as Fluo-4 AM, advances such as GCaMP mouse models can help improve the study of live calcium signaling in vivo. ${ }^{40}$

\section{Endothelial Calcium in Leukocyte Extravasation}

Changes in barrier function and vascular permeability are intimately connected with inflammation. Inflammatory disease states result in local cytokine and chemokine production, which, in addition to facilitating vascular leak, can also cause the recruitment of leukocyte subtypes to eliminate the inflammatory stimulus and initiate tissue repair. Moreover, endothelial calcium signaling is also important for facilitating many of the steps in the leukocyte extravasation cascade, including leukocyte rolling, adhesion, TEM, and ultimately pore closure (Figure 2B).

Calcium signaling also affects endothelial transcriptional activity and proinflammatory gene expression. Quinlan et $\mathrm{al}^{41}$ demonstrated that substance $\mathrm{P}$, a proinflammatory peptide which can be secreted by nerves and leukocytes, elicits an intracellular $\mathrm{Ca}^{2+}$ signal. The resultant $\mathrm{Ca}^{2+}$ mobilization then activates the transcription factor nuclear factor of activated T-cells (NFAT). NFAT subsequently binds to regulatory regions of the intercellular adhesion molecule 1 (ICAMI) gene causing induction of ICAM-1 expression (Figure 1). Work by Funk et $\mathrm{al}^{42}$ also suggests that an increase in intracellular calcium leading to NFAT activation can also induce vascular cell adhesion 1 (VCAM1) expression under proinflammatory conditions.

Fast rolling is the earliest leukocyte-endothelial cell interaction leading up to TEM. Here endothelial E-selectin engagement leads to an endothelial cell calcium influx. ${ }^{43}$ The next step, leukocyte arrest, is mediated by both VCAM-1 and ICAM-1. In vitro activation of VCAM-1 and ICAM-1 by crosslinking leads to an increase in endothelial cytosolic free calcium. ${ }^{44,45}$ It has also been shown that lymphocyte binding to endothelial VCAM-1 initiates an endothelial cell calcium influx and stimulates Rac1 downstream. $^{46}$ Activating endothelial ICAM-1 in rat brain endothelial cell lines resulted in phospholipase $\mathrm{C}$ phosphorylation and subsequent increases in both inositol phosphate production and intracellular calcium concentrations. Furthermore, inhibiting PKC activity and chelating intracellular calcium also reduced T-lymphocyte TEM significantly. ${ }^{47}$ Finally, an endothelial calcium influx also occurs during leukocyte TEM. Preventing an increase in intracellular endothelial cell influx prohibited neutrophil migration across endothelial cell monolayers. ${ }^{8,48}$

Specifically, downstream of leukocyte platelet endothelial cell adhesion molecule (PECAM)-endothelial cell PECAM homophilic interactions, TRPC6 mediates the endothelial calcium influx required for TEM. ${ }^{8}$ TRPC6 co-localizes with PECAM to surround leukocytes during TEM. Knockdown of endogenous TRPC6 or expression of a dominant negative TRPC6 arrested neutrophils above the endothelial monolayer. Furthermore, a neutrophil TEM blockade due to antiPECAM antibody could be overcome with Hyp9, an agonist selective for TRPC6. In addition, an in vivo model of murine dermatitis found that TRPC6 knockout mice reconstituted with wild-type bone marrow had a significant defect in TEM, further supporting that endothelial cell TRPC6mediated calcium influx is crucial for inflammation. The final step of TEM requires closing of the endothelial transmigration pores. F-actin contraction plays a role in this process, and it has been hypothesized that this may also be a calcium-dependent process, further demonstrating a role for calcium in facilitating inflammation. ${ }^{49}$

Despite conclusive evidence demonstrating that endothelial calcium influx is required to support TEM, there are few reports regarding the resultant, downstream signaling events. Kielbassa et $\mathrm{al}^{50}$ found that monocyte transmigration is reduced after treating human microvascular endothelial cells with triflouperazine, a CaM antagonist. Saito et $\mathrm{al}^{51}$ suggested that MLCK may be involved because pretreatment of human umbilical vein endothelial cells with the MLCK inhibitor ML-9 reduced MLC phosphorylation and reduced neutrophil migration. However, outside these two reports, there is no literature delineating how the endothelial calcium influx mediates downstream events during TEM. Recently, it was found that endothelial IQ-motif containing GTPase-activating protein 1 is required for leukocyte TEM, and the actin-binding calponin homology domain and the IQ motif domains are key for mediating this function. ${ }^{52,53}$ The IQ motif domain has been previously reported to bind $\mathrm{CaM}$ in other contexts, but this interaction and its role in TEM have not yet been explored. ${ }^{54}$

Regional heterogeneity of endothelial cells and the uncertainty about which types of ion channels are present in endothelial cell subsets also affect the studies of calcium signaling in the multistep inflammatory cascade. For example, inducing calcium entry into alveolar septal endothelial cells by two different signaling pathways leads to $\mathrm{Ca}^{2+}$ entry with and without surface expression of $\mathrm{P}$ selectin. ${ }^{55}$ This finding again suggests a need for studying context-dependent endothelial calcium signaling.

\section{Conclusions}

Calcium signaling in endothelial cells is a key regulatory mechanism for barrier function and inflammation. Calcium channels and downstream $\mathrm{Ca}^{2+}$-binding proteins, such as phospholipase $\mathrm{C}, \mathrm{CaM}$, and others, are key for mediating increases in permeability in response to inflammatory mediators, such as thrombin, histamine, and bradykinin. Furthermore, a calcium influx is involved in a number of steps in the inflammatory cascade, including leukocyte rolling, arrest, and TEM. Nonetheless, a great deal is left to be explored regarding endothelial calcium signaling. 
Questions remain regarding the events that occur downstream of the calcium influx during TEM, how these pathways vary in different vascular beds and in different disease states, and ultimately how these mechanisms can be modulated for therapeutic potential.

\section{References}

1. Clapham DE: Calcium signaling. Cell 2007, 131:1047-1058

2. Tiruppathi C, Minshall RD, Paria BC, Vogel SM, Malik AB: Role of $\mathrm{Ca} 2+$ signaling in the regulation of endothelial permeability. Vascul Pharmacol 2002, 39:173-185

3. Berridge MJ, Bootman MD, Roderick HL: Calcium signalling: dynamics, homeostasis and remodelling. Nat Rev Mol Cell Biol 2003, 4:517-529

4. Tran QK, Ohashi K, Watanabe H: Calcium signalling in endothelial cells. Cardiovasc Res 2000, 48:13-22

5. Tiruppathi C, Ahmmed GU, Vogel SM, Malik AB: Ca2+ signaling, TRP channels, and endothelial permeability. Microcirculation 2006, 13:693-708

6. Stolwijk JA, Zhang X, Gueguinou M, Zhang W, Matrougui K, Renken C, Trebak M: Calcium signaling is dispensable for receptor regulation of endothelial barrier function. J Biol Chem 2016, 291: 22894-22912

7. Hofmann T, Obukhov AG, Schaefer M, Harteneck C, Gudermann T, Schultz G: Direct activation of human TRPC6 and TRPC3 channels by diacylglycerol. Nature 1999, 397:259-263

8. Weber EW, Han F, Tauseef M, Birnbaumer L, Mehta D, Muller WA: TRPC6 is the endothelial calcium channel that regulates leukocyte transendothelial migration during the inflammatory response. J Exp Med 2015, 212:1883-1899

9. Bagur R, Hajnoczky G: Intracellular $\mathrm{Ca}(2+)$ sensing: its role in calcium homeostasis and signaling. Mol Cell 2017, 66:780-788

10. Lewit-Bentley A, Rety S: EF-hand calcium-binding proteins. Curr Opin Struct Biol 2000, 10:637-643

11. Swulius MT, Waxham MN: $\mathrm{Ca}(2+) /$ calmodulin-dependent protein kinases. Cell Mol Life Sci 2008, 65:2637-2657

12. Toutenhoofd SL, Foletti D, Wicki R, Rhyner JA, Garcia F, Tolon R, Strehler EE: Characterization of the human CALM2 calmodulin gene and comparison of the transcriptional activity of CALM1, CALM2 and CALM3. Cell Calcium 1998, 23:323-338

13. Jurado LA, Chockalingam PS, Jarrett HW: Apocalmodulin. Physiol Rev 1999, 79:661-682

14. Adams PJ, Ben-Johny M, Dick IE, Inoue T, Yue DT: Apocalmodulin itself promotes ion channel opening and $\mathrm{Ca}(2+)$ regulation. Cell 2014, 159:608-622

15. Pitt GS, Zuhlke RD, Hudmon A, Schulman H, Reuter H, Tsien RW: Molecular basis of calmodulin tethering and $\mathrm{Ca} 2+$-dependent inactivation of L-type Ca2+ channels. J Biol Chem 2001, 276: 30794-30802

16. Borbiev T, Verin AD, Shi S, Liu F, Garcia JG: Regulation of endothelial cell barrier function by calcium/calmodulin-dependent protein kinase II. Am J Physiol Lung Cell Mol Physiol 2001, 280: L983-L990

17. Wang Z, Ginnan R, Abdullaev IF, Trebak M, Vincent PA, Singer HA: Calcium/Calmodulin-dependent protein kinase II delta 6 (CaMKIIdelta6) and RhoA involvement in thrombin-induced endothelial barrier dysfunction. J Biol Chem 2010, 285:21303-21312

18. Donato R, Cannon BR, Sorci G, Riuzzi F, Hsu K, Weber DJ, Geczy CL: Functions of S100 proteins. Curr Mol Med 2013, 13: 24-57

19. Wang L, Luo H, Chen X, Jiang Y, Huang Q: Functional characterization of S100A8 and S100A9 in altering monolayer permeability of human umbilical endothelial cells. PLoS One 2014, 9:e90472
20. Gerke V, Creutz CE, Moss SE: Annexins: linking Ca2+ signalling to membrane dynamics. Nat Rev Mol Cell Biol 2005, 6: 449-461

21. Luo M, Flood EC, Almeida D, Yan L, Berlin DA, Heerdt PM, Hajjar KA: Annexin A2 supports pulmonary microvascular integrity by linking vascular endothelial cadherin and protein tyrosine phosphatases. J Exp Med 2017, 214:2535-2545

22. Heyraud S, Jaquinod M, Durmort C, Dambroise E, Concord E, Schaal JP, Huber P, Gulino-Debrac D: Contribution of annexin 2 to the architecture of mature endothelial adherens junctions. Mol Cell Biol 2008, 28:1657-1668

23. Rodrigues SF, Granger DN: Blood cells and endothelial barrier function. Tissue Barriers 2015, 3:e978720

24. Tauseef M, Knezevic N, Chava KR, Smith M, Sukriti S, Gianaris N, Obukhov AG, Vogel SM, Schraufnagel DE, Dietrich A, Birnbaumer L, Malik AB, Mehta D: TLR4 activation of TRPC6dependent calcium signaling mediates endotoxin-induced lung vascular permeability and inflammation. J Exp Med 2012, 209: $1953-1968$

25. Singh I, Knezevic N, Ahmmed GU, Kini V, Malik AB, Mehta D: Galphaq-TRPC6-mediated Ca2+ entry induces RhoA activation and resultant endothelial cell shape change in response to thrombin. J Biol Chem 2007, 282:7833-7843

26. Graham S, Ding M, Ding Y, Sours-Brothers S, Luchowski R, Gryczynski Z, Yorio T, Ma H, Ma R: Canonical transient receptor potential 6 (TRPC6), a redox-regulated cation channel. J Biol Chem 2010, 285:23466-23476

27. Leung PC, Cheng KT, Liu C, Cheung WT, Kwan HY, Lau KL, Huang Y, Yao X: Mechanism of non-capacitative Ca2+ influx in response to bradykinin in vascular endothelial cells. J Vasc Res 2006, 43:367-376

28. Sandoval R, Malik AB, Naqvi T, Mehta D, Tiruppathi C: Requirement for $\mathrm{Ca} 2+$ signaling in the mechanism of thrombin-induced increase in endothelial permeability. Am J Physiol Lung Cell Mol Physiol 2001, 280:L239-L247

29. Popovic M, Smiljanic K, Dobutovic B, Syrovets T, Simmet T, Isenovic ER: Thrombin and vascular inflammation. Mol Cell Biochem 2012, 359:301-313

30. Vogel SM, Gao X, Mehta D, Ye RD, John TA, Andrade-Gordon P, Tiruppathi $\mathrm{C}$, Malik AB: Abrogation of thrombin-induced increase in pulmonary microvascular permeability in PAR-1 knockout mice. Physiol Genomics 2000, 4:137-145

31. Tauseef M, Farazuddin M, Sukriti S, Rajput C, Meyer JO, Ramasamy SK, Mehta D: Transient receptor potential channel 1 maintains adherens junction plasticity by suppressing sphingosine kinase 1 expression to induce endothelial hyperpermeability. FASEB J 2016, 30:102-110

32. Rabiet MJ, Plantier JL, Rival Y, Genoux Y, Lampugnani MG, Dejana E: Thrombin-induced increase in endothelial permeability is associated with changes in cell-to-cell junction organization. Arterioscler Thromb Vasc Biol 1996, 16:488-496

33. Sandoval R, Malik AB, Minshall RD, Kouklis P, Ellis CA, Tiruppathi $\mathrm{C}$ : $\mathrm{Ca}(2+)$ signalling and $\mathrm{PKCalpha}$ activate increased endothelial permeability by disassembly of VE-cadherin junctions. J Physiol 2001, 533:433-445

34. van Nieuw Amerongen GP, Draijer R, Vermeer MA, van Hinsbergh VW: Transient and prolonged increase in endothelial permeability induced by histamine and thrombin: role of protein kinases, calcium, and RhoA. Circ Res 1998, 83:1115-1123

35. Winter MC, Kamath AM, Ries DR, Shasby SS, Chen YT, Shasby DM: Histamine alters cadherin-mediated sites of endothelial adhesion. Am J Physiol 1999, 277:L988-L995

36. Sidibe A, Imhof BA: VE-cadherin phosphorylation decides: vascular permeability or diapedesis. Nat Immunol 2014, 15:215-217

37. Eriksson EE, Karlof E, Lundmark K, Rotzius P, Hedin U, Xie X: Powerful inflammatory properties of large vein endothelium in vivo. Arterioscler Thromb Vasc Biol 2005, 25:723-728 
38. Stevens T: Functional and molecular heterogeneity of pulmonary endothelial cells. Proc Am Thorac Soc 2011, 8:453-457

39. Taylor MS, Francis M: Decoding dynamic $\mathrm{Ca}(2+)$ signaling in the vascular endothelium. Front Physiol 2014, 5:447

40. Duza T, Sarelius IH: Localized transient increases in endothelial cell $\mathrm{Ca} 2+$ in arterioles in situ: implications for coordination of vascular function. Am J Physiol Heart Circ Physiol 2004, 286:H2322-H2331

41. Quinlan KL, Naik SM, Cannon G, Armstrong CA, Bunnett NW, Ansel JC, Caughman SW: Substance P activates coincident NF-ATand NF-kappa B-dependent adhesion molecule gene expression in microvascular endothelial cells through intracellular calcium mobilization. J Immunol 1999, 163:5656-5665

42. Funk SD, Finney AC, Yurdagul A Jr, Pattillo CB, Orr AW: EphA2 stimulates VCAM-1 expression through calcium-dependent NFAT1 activity. Cell Signal 2018, 49:30-38

43. Lorenzon P, Vecile E, Nardon E, Ferrero E, Harlan JM, Tedesco F, Dobrina A: Endothelial cell E- and P-selectin and vascular cell adhesion molecule-1 function as signaling receptors. J Cell Biol 1998, 142:1381-1391

44. Clayton A, Evans RA, Pettit E, Hallett M, Williams JD, Steadman R: Cellular activation through the ligation of intercellular adhesion molecule-1. J Cell Sci 1998, 111:443-453

45. Ricard I, Payet MD, Dupuis G: Clustering the adhesion molecules VLA-4 (CD49d/CD29) in Jurkat T cells or VCAM-1 (CD106) in endothelial (ECV 304) cells activates the phosphoinositide pathway and triggers Ca2 + mobilization. Eur J Immunol 1997, 27:1530-1538

46. Cook-Mills JM, Johnson JD, Deem TL, Ochi A, Wang L, Zheng Y: Calcium mobilization and Rac1 activation are required for VCAM-1 (vascular cell adhesion molecule-1) stimulation of NADPH oxidase activity. Biochem J 2004, 378:539-547

47. Etienne-Manneville S, Manneville JB, Adamson P, Wilbourn B, Greenwood J, Couraud PO: ICAM-1-coupled cytoskeletal rearrangements and transendothelial lymphocyte migration involve intracellular calcium signaling in brain endothelial cell lines. J Immunol 2000, 165:3375-3383

48. Huang AJ, Manning JE, Bandak TM, Ratau MC, Hanser KR, Silverstein SC: Endothelial cell cytosolic free calcium regulates neutrophil migration across monolayers of endothelial cells. J Cell Biol 1993, 120:1371-1380

49. Heemskerk N, Schimmel L, Oort C, van Rijssel J, Yin T, Ma B, van Unen J, Pitter B, Huveneers S, Goedhart J, Wu Y, Montanez E, Woodfin A, van Buul JD: F-actin-rich contractile endothelial pores prevent vascular leakage during leukocyte diapedesis through local RhoA signalling. Nat Commun 2016, 7:10493

50. Kielbassa K, Schmitz C, Gerke V: Disruption of endothelial microfilaments selectively reduces the transendothelial migration of monocytes. Exp Cell Res 1998, 243:129-141

51. Saito H, Minamiya Y, Kitamura M, Saito S, Enomoto K, Terada K, Ogawa J: Endothelial myosin light chain kinase regulates neutrophil migration across human umbilical vein endothelial cell monolayer. J Immunol 1998, 161:1533-1540

52. Sullivan DP, Dalal PJ, Jaulin F, Sacks DB, Kreitzer G, Muller WA Endothelial IQGAP1 regulates leukocyte transmigration by directing the LBRC to the site of diapedesis. J Exp Med 2019, 216:2582-2601

53. Nakhaei-Nejad M, Zhang QX, Murray AG: Endothelial IQGAP1 regulates efficient lymphocyte transendothelial migration. Eur J Immunol 2010, 40:204-213

54. Li Z, Sacks DB: Elucidation of the interaction of calmodulin with the IQ motifs of IQGAP1. J Biol Chem 2003, 278:4347-4352

55. Wu SW, Jian MY, Xu YC, Zhou C, Al-Mehdi AB, Liedtke W, Shin HS, Townsley MI: Ca2+ entry via alpha(1G) and TRPV4 channels differentially regulates surface expression of P-selectin and barrier integrity in pulmonary capillary endothelium. Am J Physiol Lung Cell Mol Physiol 2009, 297:L650-L657 